Transaction

Transaction

(Received February 12, 1992)

\title{
EFFECTS OF THE SUBSTITUENT DISTRIBUTION ALONG THE MAIN CHAIN ON SOME PROPERTIES OF o-(2,3-DIHYDROXYPROPYL) CELLULOSE
}

\author{
Takeshi Fukuda, Takaya Sato*1, and Takeaki Miyamoto \\ Institute for Chemical Research, Kyoto University, Uji, Kyoto, 611 Japan
}

\begin{abstract}
A novel polyhydroxy polymer, o-(2,3-dihydroxypropyl) cellulose (DHPC) was synthesized by reaction of cellulose with 2,3-epoxy-1-propanol (glycidol). The reaction was carried out in both homogeneous and heterogeneous media, which resulted in two types of DHPCs, coded DH and HDH, respec. tively. They were compared with each other with respect to structural and physicochemical properties, such as relation between molar substitution-degree of substitution, substituent distribution within anhydroglucose unit, water solubility, aqueous solution viscosity, surface activation effect and thermal and dielectric properties of cyanoethyl DHPCs. Significant differences between the two types of DHPCs were observed in all these properties, which were discussed in terms of the difference in the substituent distribution along the cellulose chain.
\end{abstract}

\section{INTRODUCTION}

$o$-(2,3-Dihydroxypropyl) cellulose (DHPC) is a polyhydroxy cellulose derivative produced by reacting cellulose with 2,3-epoxy-1-propanol (glycidol) or 1-chloro-2,3-propandiol. Under a suitable reaction condition, derivatives with more than three hydroxyl groups per glucose unit can be obtained (Fig. 1). DHPC is a novel polymer (1) about which only a few reports have been published: e.g., Hogen-esh et al. re-

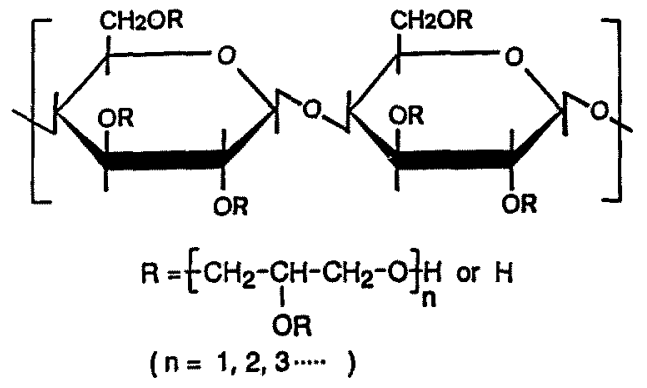

Fig. 1. Chemical structure of $O-(2,3$-dihydroxypropyl) cellulose (DHPC).

${ }^{*}$ Present address; Nisshinbo Industries Inc. Tokyo Research Center, Nishiarai-Sakaecho, Adachi-ku, Tokyo, 123 Japan

*1 現在の所属; 日清紡績(株)東京研究センター, 東京 都足立区西新井栄町 ported about the graft polymerization of acrylamide on DHPC [2] and we reported about the synthesis of a highly dielectric polymer by the cyanoethylation of DHPC $[3,4]$.

Structurally, DHPC is different from other cellulose derivatives such as methyl cellulose and cellulose ace. tate in that the substituents can be oligo-dihydroxy. propyl chains of differing lengths $(n \geq 1)$. Therefore, the total number of glycidol units incorporated into anhydroglucose unit, i.e., the molar substitution (MS) as well as the degree of substitution (DS) are important parameters to characterize their structure. It is also known that properties of cellulose derivatives depend not only on DS and MS but also on the substituent distribution along the cellulose chain. Differences in the uniformity of the distribution can result in materials differing in performance properties, even when the DS and MS values are similar. When the hydroxyalkylation is carried out in a heterogeneous phase in which the starting material as well as the product remain in partilly crystalline states, one will obtain a derivative having a non-uniform distribution of the substituents along the chain, because crystalline or insoluble regions of a cellulosic material will have a lower reactivity than amorphous or soluble regions. On the other hand, in a homogeneous reaction condition which makes use of nonaqueous solvent sys- 
tems such as LiCl-dimethylacetamide and $\mathrm{SO}_{2}-$ dimethylsulfoxide-diethylamine, the product will have a more uniform substituent distribution than in a heterogeneous system.

In this work, we have prepared two types of DHPC samples using homogeneous and heterogeneous reac. tion conditions, and compared them with respect to their structural and physicochemical properties. The results will be discussed in relation to the substituent distributions of the two types of DHPCs.

\section{EXPERIMENTAL}

\subsection{Materials}

Cellulose sample, Avicel PH-101 (DP $=210$, Asahi Chemical Industry) was dried at $105^{\circ} \mathrm{C}$ for $24 \mathrm{~h}$ before use. 2,3-Epoxy-1-propanol (glycidol), purchased from Nacalai Tesque, was purified by vacuum distillation before use. All other chemicals were of reagent grade and used without further purification.

\subsection{Preparation of o-(2,3-Dihydroxypropyl) cellulose (DHPC)}

\subsubsection{Homogeneous Reaction System (DH-series)}

The DHPC polymers with different molar substitution (MS) were prepared by the homogeneous reaction of cellulose with glycidol in a $10 \mathrm{wt} . \% \mathrm{LiCl}$-dimethylacetamide (DMAc) solvent, as described in a previous paper [4].

\subsubsection{Heterogeneous Reaction System (HDH-series)}

Five grams of cellulose powder was suspended in $70 \mathrm{~mL}$ of isopropyl alcohol and cooled to $0{ }^{\circ} \mathrm{C}$, to which $10 \mathrm{~mL}$ of $18.5 \mathrm{wt}$. \% aqueous sodium hydroxide solution was added, and the system was vigorously stirred for $1 \mathrm{~h}$ at $0^{\circ} \mathrm{C}$. The mixture was then neut ralized with $2.40 \mathrm{~g}$ of acetic acid to adjust the molar ratio of $\mathrm{NaOH} /$ anhydroglucose unit (AHG) to 0.2 , and kept standing for $12 \mathrm{~h}$ at $0{ }^{\circ} \mathrm{C}$. To this cellulose solution, an isopropyl alcohol solution of glycidol was added dropwise over a period of $12 \mathrm{~h}$ at $50^{\circ} \mathrm{C}$ in order to prevent the homopolymerization of glycidol. The mixture was stirred at $50^{\circ} \mathrm{C}$ for another $24 \mathrm{~h}$. During the reaction, the cellulose powder did not swell, remaining in a powder form. After the mixture was cooled, the product was neutralized with acetic acid, filtered, and washed with isopropyl alcohol. The product was dissolved in deionized water, dialyzed, and freeze-dried. The results of the heterogeneous
Table 1 Results of the Dihydroxypropylation in a Heterogeneous Medium

\begin{tabular}{|c|c|c|c|}
\hline & \multirow{2}{*}{$\begin{array}{l}\text { Sample } \\
\text { code }\end{array}$} & {$[G L Y]^{a)}$} & \multirow{2}{*}{$\mathrm{MS}^{\mathrm{b})}$} \\
\hline & & [AHG] & \\
\hline & HDH-1 & 20 & 1.5 \\
\hline & $\mathrm{HDH}-2 \mathrm{~A}$ & 28 & 2.3 \\
\hline & $\mathrm{HDH}-2 \mathrm{~B}$ & 28 & 2.5 \\
\hline & $\mathrm{HDH}-3$ & 30 & 2.9 \\
\hline & $\mathrm{HDH}-4$ & 45 & 3.8 \\
\hline & $\mathrm{HDH}-5$ & 53 & 5.5 \\
\hline $\begin{array}{l}\text { a) } \\
\text { b) }\end{array}$ & $\begin{array}{l}\text { Molar rati } \\
\text { Estimated }\end{array}$ & Lant & nit]. \\
\hline
\end{tabular}

dihydroxypropylation are summarized in Table 1.

In what follows, the samples prepared by heterogeneous and homogeneous reaction systems will be designated as HDH and DH, respectively, the number attached to each sample code denoting its approximate MS value.

\subsection{Cyanoethylation of DHPC}

Cyanoethylation of DHPC was carried out by the Michael condensation with acrylonitrile using sodium hydroxide as catalyst. A detailed preparation procedure was described previously [4]. Samples used for the dielectric measurements were further purified to remove ionic impurities by dialyzing their acetone solutions against deionized water [3].

\subsection{Measurements}

${ }^{13} \mathrm{C}-\mathrm{NMR}$ spectra were obtained at $80^{\circ} \mathrm{C}$ on a JEOL $\mathrm{GX}-400$ spectrometer operating at $100.8 \mathrm{MHz}$ in the proton noise-decoupled mode, by using a $10 \mathrm{~mm}$ probe and deuterated dimethylsulfoxide $\left(D M S O-\mathrm{d}_{6}\right)$ as solvent. Other measurement conditions were same as described before [4].

$\mathrm{X}$-ray diffraction patterns were recorded on a Rigaku-Denki Model RINT-1500 X-ray diffractometer by use of a disk-shaped sample ( $40 \mathrm{mg}$ ) prepared by applying compression at a pressure of $100 \mathrm{~kg} / \mathrm{cm}^{2}$. The equatorial diffraction patterns were recorded in a $2 \theta$ range of $5^{\circ}$ to $40^{\circ}$ with a $\mathrm{Cu}-\mathrm{K} \alpha$ radiation at $40 \mathrm{kV}$ and $100 \mathrm{~mA}$. The scanning rate and the slit width were $1 \%$ min and $0.8 \mathrm{~mm}$, respectively.

Gel permeation chromatography (GPC) was performed at $25^{\circ} \mathrm{C}$ with a Toso HLC-803C high speed liquid chromatograph equipped with a differential re- 
fractometer Model RI-8. Hitachi GL-W520, GLW530, and GL-W540 columns were used with a $0.5 \%$ acetic acid solution as eluent. The weight-aver. age molecular weight was estimated on the basis of the calibration curve established with standard poly (ethylene oxide)s.

The viscosity of DHPC aqueous solution (3 wt.-\%) was measured at $25^{\circ} \mathrm{C}$ by using a rotating viscometer, Tokyo-Keiki Viscometer Type-B. The surface pressures of aqueous DHPC solutions ( 1 wt.-\%) were measured at $25^{\circ} \mathrm{C}$ on a film balance of a Wilhelmy type.

The dielectric properties of the cyanoethylated DHPC samples were examined with a Hewlett Packard $4284 \mathrm{~A}$ Precision LCR meter, at $27^{\circ} \mathrm{C}$ and $1 \mathrm{KHz}$. The thermal properties of the cyanoethylated polym. ers were studied on a Rigaku-Denki Model DSC-8230 at a constant heating / cooling rate of $10 \mathrm{~K} / \mathrm{min}$. Polarized light microscopic observations were made with a Nikon Model Optiphoto-Pol equipped with a Mettler hot stage Model FP-82 and a temperature controller Model FP-80. Detailed procedures were described previously $[3,4]$.

\section{RESULTS AND DISCUSSION}

\subsection{Preparation and Characterization of DHPCs}

Previous reports from this laboratory dealed with the preparation of DHPCs by the reaction of cellulose with glycidol in a nonaqueous, homogeneous solution with 10 wt.-\% $\mathrm{LiCl} / \mathrm{DMAc}(3,4]$. This report deals with a comparison of these DHPCs (DH series) with those (HDH series) prepared by the alkali cellulose process that provides a heterogeneous reaction medium. To prepare the HDH samples, cellulose was pretreated according to the method of Sikkema [5]: namely, cellulose was swollen in isopropyl alcohol with an adequate amount of alkali to destroy its crystallinity and convert it to a completely amorphous material. Subsequently, the system was neutralized with acetic acid to a low alkali level and kept stand. ing at $0^{\circ} \mathrm{C}$ to allow recrystallization. This process is expected to give a cellulose having microcrystalline regions more or less randomly distributed along the chain. A substitution reaction starting with this semicrystalline material would provide a derivative with a segmented-block copolymer-type structure, because

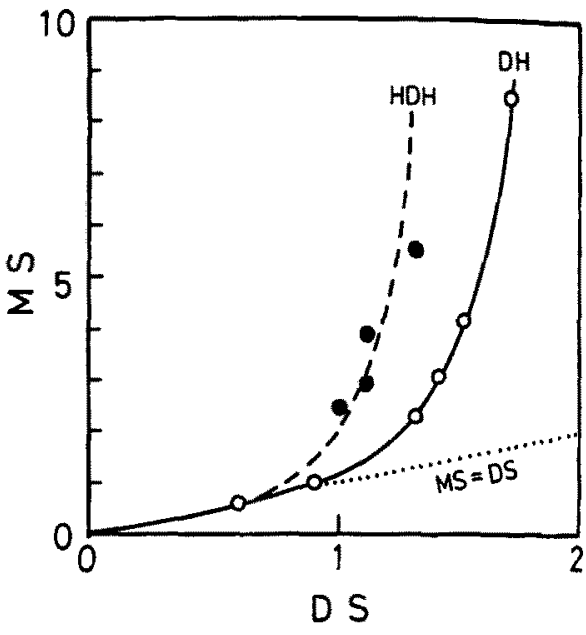

Fig. 2. Plot of MS vs. DS for DHPCs.

the crystalline regions would be more resistant to the substitution reaction than the amorphous ones. By this method. Sikkema [5] produced a carboxymethyl cellulose whose properties were distinctly different from those of the one produced by the conventional alkali cellulose method starting with an untreated cel. lulose.

Fig. 2 shows a comparison of the MS vs. DS rela. tions for the DH and HDH series of DHPCs. Regard. ing the DH samples, MS is nearly equal to DS for small value of $D S$, but it becomes exceedingly larger than DS for DS $\geq 1$, showing that in later stages of the reaction, glycidol preferentially adds to the side chains rather than to the main chain. This is essentially due to the increased number of hydroxyl groups in the side chains in later stages. However, the observed MS vs. DS relation cannot be fully described by quasi-first order kinetics (6), indicating that glycidol molecules, which were supplied to the system little by little over a long period of time (see above), became more and more difficult to reach the main chain as the side chains grew. Fig. 2 also shows that the curve for the $\mathrm{HDH}$ samples rises more steeply than the DH curve. The broken line in the figure. which was obtained by multiplying the $\mathrm{DH}$ curve by a factor 0.73 with respect to both the ordinate and abscissa scales, well reproduces the experimental points. This means that about $27 \%$ of the hydroxyl groups in the main chain is unavailable for the reac. 


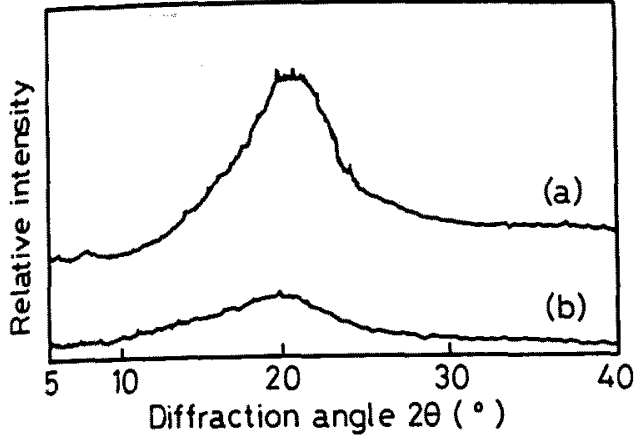

Fig. 3. X-ray diffraction patterns of (a) $\mathrm{HDH}-3$ and (b) an amorphous cellulose.

Table 2 Substituent Distribution in DHPCs

\begin{tabular}{llllll}
\hline \multirow{2}{*}{$\begin{array}{l}\text { Sample } \\
\text { code }\end{array}$} & \multirow{2}{*}{ MS } & Total DS & \multicolumn{3}{c}{ Regional DS } \\
\cline { 5 - 6 } & & & $\mathrm{C}-2$ & $\mathrm{C}-3$ & $\mathrm{C}-6$ \\
\hline $\mathrm{DH}-2$ & 2.3 & 1.3 & 0.5 & 0.2 & 0.6 \\
$\mathrm{DH}-4$ & 4.2 & 1.5 & 0.6 & 0.3 & 0.6 \\
$\mathrm{DH}-8$ & 8.5 & 1.7 & 0.6 & 0.4 & 0.7 \\
$\mathrm{HDH}-3$ & 2.9 & 1.05 & 0.5 & 0.05 & 0.5 \\
$\mathrm{HDH}-4$ & 3.8 & 1.05 & 0.4 & 0.05 & 0.6 \\
$\mathrm{HDH}-5$ & 5.5 & 1.3 & 0.6 & 0.1 & 0.6 \\
\hline
\end{tabular}

Table 3 Values of MS and DS and Water Solubility of DHPCs

\begin{tabular}{|c|c|c|c|}
\hline $\begin{array}{l}\text { Sample } \\
\text { code }\end{array}$ & MS & DS & Solubility ${ }^{\text {a) }}$ \\
\hline \multicolumn{4}{|c|}{ Homogeneous } \\
\hline $\mathrm{DH}-1 \mathrm{~A}$ & 0.6 & 0.6 & 0 \\
\hline $\mathrm{DH}-1 \mathrm{~B}$ & 1.0 & 0.9 & 0 \\
\hline $\mathrm{DH}-2$ & 2.3 & 1.3 & 0 \\
\hline $\mathrm{DH}-3$ & 3.1 & 1.4 & 0 \\
\hline $\mathrm{DH}-4$ & 4.2 & 1.5 & 0 \\
\hline $\mathrm{DH}-8$ & 8.5 & 1.7 & 0 \\
\hline \multicolumn{4}{|c|}{ Heterogeneous } \\
\hline $\mathrm{HDH}-1$ & 1.5 & n.d. ${ }^{\mathrm{b})}$ & $x$ \\
\hline HDH-2A & 2.3 & n.d. ${ }^{b)}$ & $\triangle$ \\
\hline $\mathrm{HDH}-2 \mathrm{~B}$ & 2.5 & 1.0 & $\triangle$ \\
\hline $\mathrm{HDH}-3$ & 2.9 & 1.1 & $\Delta-O$ \\
\hline $\mathrm{HDH}-4$ & 3.8 & 1.1 & 0 \\
\hline $\mathrm{HDH}-5$ & 5.5 & 1.3 & 0 \\
\hline $\begin{array}{l}\text { For } 2 \% \\
x_{\text {; insol }}\end{array}$ & \multicolumn{2}{|c|}{$x_{i}$ insoluble, $\Delta ;$ swollen } & O; soluble, \\
\hline $\begin{array}{l}\text { b) Not deter } \\
\text { NMR sol }\end{array}$ & d bec: & the $\mathrm{i}$ & ity in common \\
\hline
\end{tabular}

tion, indicating that there is about this much of crystalline regions to which glycidol is inaccessible. In fact, sample HDH-3, for example, exhibits a considerable crystallinity, as shown by the $\mathrm{X}$-ray diffraction pattern given in Fig. 3.

Table 2 shows the substituent distribution within an $\mathrm{AHG}$ unit as determined by ${ }^{13} \mathrm{C}-\mathrm{NMR}(4,7)$. For the DH samples, the DS value increases in the order of $\mathrm{C}-3<\mathrm{C}-2 \cong \mathrm{C}-6$. The same order is true with the HDH samples, but the DS of the C-3 hydroxyl group is extremely low, showing that the relative reactivity of the $\mathrm{C}-3$ position in the heterogeneous medium is much lower than in the homogeneous medium. This difference could be interpreted, at least in part, in terms of the mentioned crystallinity of the HDH samples, if we assume that the $\mathrm{C}-3$ hydroxyl groups take more part in crystallization than those at the other two positions, the latter preferentially appearing on the crystallite surfaces and hence being more easily substituted $[8,9]$.

\subsection{Properties of DHPCs in Aqueous Solution}

Table 3 shows the results of a water-solubility test carried out for the two series of DHPCs. All examined DH samples were soluble in water. Notably, even Sample DH-1A with an MS ( $\cong$ DS) as low as $0.6 \mathrm{ex}$ hibited complete solubility. On the other hand, the $\mathrm{HDH}$ sample with $\mathrm{MS}=1.5$ (HDH-1), whose DS should be about 0.9 (see Fig. 2), was insoluble, and samples HDH-2A, and $-2 \mathrm{~B}$ were only swollen but not soluble. Sample HDH-3 (DS $=1.1, \mathrm{MS}=2.9$ ) was partly soluble with a considerable portion remaining only swollen. Samples with higher DS and MS values were apparently soluble (see below). Thus, the differences in water solubility between the two series of samples are clear. The relatively low solubility of the HDH samples no doubt derives from an uneven dis. tribution of the substituents along the chain. Thus, the presence of relatively long unsubstituted segments that resist solubilization is suggested by this test.

Table 4 compares the viscosities of two apparently water-soluble samples, DH-3 and HDH-4. Despite that the two samples have nearly same GPC elution volume, the viscosity of the $3 \mathrm{wt}-\%$ aqueous solution of HDH-4 is nearly twice as large as that of DH-3. It is well known that water soluble cellulose ethers such as methyl cellulose (MC) and ethyl cellulose (EC) 
Table 4 Comparison of Solution Viscosity between DH and HDH Samples

\begin{tabular}{lcc}
\hline $\begin{array}{l}\text { Sample } \\
\text { code }\end{array}$ & $\mathrm{M}_{\mathrm{w}}^{\mathrm{a}}$ & $\begin{array}{c}\text { Viscosity } \\
(\mathrm{cp})\end{array}$ \\
\hline $\mathrm{DH}-3$ & 41000 & 40 \\
$\mathrm{HDH}-4$ & 39000 & 75 \\
\hline a) & Estimated by a poly (ethylene oxide) -calibrated GPC \\
analysis in 0.5\% acetic acid solution. \\
b) Measured for a 3 wt.-\% aqueous solution by a rotat- \\
ing viscometer.
\end{tabular}

Table 5 Surface Tensions of 1 wt.-\% DHPC Aqueous Solutions $\left(25^{\circ} \mathrm{C}\right)$

\begin{tabular}{lc}
$\begin{array}{l}\text { Sample } \\
\text { code }\end{array}$ & $\begin{array}{c}\text { Surface tension } \\
(\mathrm{mN} / \mathrm{m})\end{array}$ \\
\hline $\mathrm{H}_{2} \mathrm{O}$ & 72.3 \\
$\mathrm{DH}-2$ & 71.3 \\
$\mathrm{DH}-4$ & 70.5 \\
$\mathrm{DH}-8$ & 71.4 \\
$\mathrm{HDH}-3$ & 62.8 \\
$\mathrm{HDH}-4$ & 66.1 \\
$\mathrm{HDH}-5$ & 65.8 \\
\hline
\end{tabular}

show thermally reversible sol-gel transitions in aqueous solution. Commercial water soluble MC pro. ducts, usually prepared in a heterogeneous reaction condition, will have trimethyl glucose sequences in the chain. It has been considered that the insoluble trimethyl glucose sequences act as "crosslinking loci" on warming [7]. However, our water-soluble DHPCs of both types showed no gelation even when the temperature was raised to $95^{\circ} \mathrm{C}$. The observed large difference between the viscosities of the two DHPCs again suggests the presence of relatively long unsub stituted sequences in the HDH sample, which perhaps promote aggregation of the portions of the molecules acting as a kind of crosslinking loci upon increasing concentration.

Another interesting difference between the two types of DHPCs was found in the interfacial properties of their aqueous solutions. Surface tension data are summarized in Table 5 . The aqueous solutions of the DH samples have a similar surface tension to water, while those of the $\mathrm{HDH}$ samples have a lower sur.

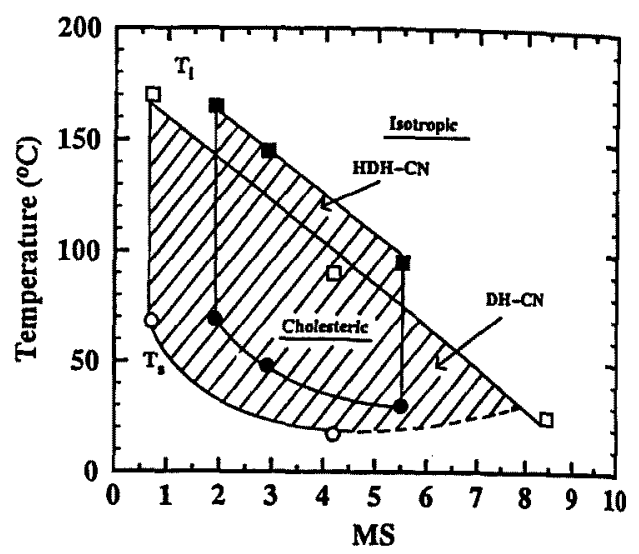

Fig. 4. Phase transition temperatures of fully cyanoethylated DHPCs as a function of MS: (OD), HDH-polymers; (O $\mathrm{O}$ ), DH-polymers.

face tension. In general, amphiphilic polymers have an ability to decrease the surface tension, and this abil. ity strongly depends on the hydrophilic-hydrophobic (lipophilic) balance. In this regard, the DH-series of samples would have little surface activity, because of the even distribution of the substituents along the chain, while the HDH series of samples would have some amphiphilicity, albeit weak: namely, the substituted and unsubstituted sequences have different hyd. rophilicity and would behave, if they are sufficiently long, as hydrophilic and hydrophobic segments respectively. This amphiphilicity is considered to be responsible for the observed surface activation effects of the HDH samples.

\subsection{Thermal and Dielectric Properties of Cyanoethylated DHPCs}

We previously studied the thermal and dielectric properties of cyanoethyl derivatives of DHPCs of the DH type $[3,4]$. Those derivatives were found to be characterized by low glass transition temperatures, $\mathrm{T}_{\mathrm{g}}\left(-30--40^{\circ} \mathrm{C}\right)$, extremely high dielectric con stants $(20-30$ at room temperature), and the occurr ence of cholesteric liquid crystal (LC) phase over a wide temperature range. Fig. 4 shows the phase dia gram of those derivatives (DH-CNs). In the figure, $T_{i}$ denotes the anisotropic-to-isotropic transition temperature, and $T_{S}$ denotes the temperature at which a small endothermic discontinuity of the DSC heating curve was observed. This temperature $T_{s}$, well above 
Table 6 Dielectric Constant $\left(\varepsilon^{\prime}\right)$ and Dielectric Loss Tangent ( $\left.\tan \delta\right)$ of Fully Cyanoethy. lated DHPCs

\begin{tabular}{lccl}
$\begin{array}{l}\text { Sample } \\
\text { code }\end{array}$ & $\begin{array}{c}\text { Dielectric } \\
\text { constant } \\
\left.\left(\varepsilon^{\prime}\right)^{\mathrm{a}}\right)\end{array}$ & $\begin{array}{c}\text { Dielectric } \\
\text { loss tangent } \\
(\tan \delta)^{\mathrm{a})}\end{array}$ & \multicolumn{1}{c}{ Appearance $^{\mathrm{b})}$} \\
\hline $\mathrm{DH}-1-\mathrm{CN}$ & 18 & 0.01 & hard-waxy \\
$\mathrm{DH}-4-\mathrm{CN}$ & 31 & 0.04 & liquid crystalline (cholesteric) \\
$\mathrm{DH}-8-\mathrm{CN}$ & 26 & 0.13 & isotropic liquid \\
$\mathrm{HDH}-2-\mathrm{CN}$ & 28 & 0.05 & hard-waxy \\
$\mathrm{HDH}-3-\mathrm{CN}$ & 27 & 0.06 & hard-waxy \\
$\mathrm{HDH}-5-\mathrm{CN}$ & 30 & 0.04 & hard-waxy \\
\hline a) At $1 \mathrm{kHz}$ and $27^{\circ} \mathrm{C}$. & & \\
b) At room temperature. & &
\end{tabular}

$T_{g}$ was interpreted as being the one at which certain reorganization of the semirigid $\mathrm{DH}-\mathrm{CN}$ molecules to a liquid crystalline order took place [3]. Between $T_{g}$ and $\mathrm{T}_{s}$, the polymers had a hard-waxy appearance, but above $T_{s}$, they showed obvious fluidity and $a \mathrm{li}$ quid crystalline birefringence.

The fully cyanoethylated DHPCs of the HDH type (HDH-CN) prepared here have somewhat different properties. They also form a cholesteric LC, but the temperature region of their LC phase is somewhat higher than that of DH-CN, when compared at a common value of MS (Fig. 4). Owing to this, all HDH-CN samples examined here have a hard-waxy, solidlike appearance at, and even significantly above, the room temperature. Also importantly, the dielectric constants of $\mathrm{HDH}-\mathrm{CNs}$ are as high as those of DH-CNs, as shown in Table 6 . Clearly, the substituent distribution has some effect on the phase behavior of the polymers but no effect on their dielectric constants. The magnitude of $\varepsilon^{\prime}$ is governed essentially by MS or the number of cyanoethyl groups introduced in the DHPC chain.

\section{CONCLUSION}

DHPCs prepared in a homogeneous (DH-series) and a heterogeneous (HDH-series) reaction medium were compared with respect to some structural and physicochemical properties. The MS vs. DS relation, the $\mathrm{X}$ ray diffraction pattern, and the ${ }^{13} \mathrm{C}-\mathrm{NMR}$ data all suggest the existence of crystallizable, hence relatively long, unsubstituted sequences in the HDH polymers. Generally, the HDH polymers have a poorer solubil- ity, a larger solution viscosity (at a high concentration), and a higher surface activity, in an aqueous medium, than the comparable DH polymers. These differences can be attributed to the difference in the substituent distribution between the two types of derivatives. Fully cyanoethylated $\mathrm{HDH}$ generally have a liquid crystal phase at temperatures somewhat higher than those of the $\mathrm{DH}$ counterparts. Both types of cyanoethylated derivatives have extraordinarily high dielectric constants.

\section{REFERENCES}

1. Y. -X. Zhang, J. C. Chen, D. Patil, G. B. Butler, and T. E. Hogen-esch, J. Macromol. Sci., Chem., A25(8), 955 (1988)

2. Y. -X. Zhang, J. C. Chen, D. Patil, G. B. Butler, and T. E. Hogen-esch, J. Macromol. Sci., Chem., A25(8), 971 (1988)

3. T. Sato, Y. Tsujii, Y. Kita, T. Fukuda, and T. Miyamoto, Macromolecules, 24, 4691 (1991)

4. T. Sato, Y. Tsujii, M. Minoda, Y. Kita, and T. Miyamoto, Makromol. Chem., 193, 647 (1992)

5. D. J. Sikkema and H. Jannsen, Macromolecules, 22, 364 (1989)

6. T. Fukuda, unpublished calculations

7. S. Takahashi, T. Fujimoto, T. Miyamoto, and H. Inagaki, J. Polym. Sci., Polym. Chem. Ed., 25, 987 (1987)

8. A. Isogai, A. Ishizu, and J. Nakano, Sen'i Gakkaishi, 40, T-504 (1984)

9. K. Kimura, T. Shigemura, M. Kubo, and Y. Maru, Makromol. Chem., 186, 61 (1985) 